\title{
Modeling Multi-party Web-based Business Collaborations
}

\author{
Lai Xu and Sjaak Brinkkemper \\ Institute of Information and Computing Sciences, \\ Utrecht University, 3584CH Utrecht, \\ The Netherlands \\ email: $\{$ L.Xu,S.Brinkkemper $\} @$ cs.uu.nl
}

\begin{abstract}
To remain competitive, enterprises have to mesh their business processes with their customers, suppliers and business partners. Increasing collaboration includes not only a global multi-national enterprise, but also an organization with its relationship to and business processes with its business partners. Standards and technologies permit business partners to exchange information, collaboration and carry out business transaction in a pervasive Web environment. There is however still very limited research activity on modeling multi-party Web-based business collaboration underlying semantics. In this paper, we demonstrate that an in-house business process has been gradually outsourced to third-parties and analyze how task delegations cause commitments between multiple business parties. Finally we provide process semantics for modeling multi-party Web-based collaborations.
\end{abstract}

\section{Introduction}

In the modern business world, we see that explicit structural collaboration between organizations is becoming more and more important. This is reflected in the emergence of tightly-coupled supply chains, the service outsourcing paradigm, 
complex co-makerships, etceteras. Collaboration is not limited by geographical proximity, but increasingly of an international character. As a result of this, explicit multi-party business coordinations are becoming global. The need for a multi-party collaboration model for a business process is thus becoming evident.

In the rest of this paper, we first elaborate how an in-house business process has been gradually outsourced in Section 2. In Section 3, we define our modeling language for multi-party business collaborations. We evaluate relate work in this area in Section4. The paper concludes with an summary and directions for further research in Section 5.

\section{Multi-party Business Collaborations and Outsource}

We provide a car insurance case for explaining how a car insurance business is gradually outsourced and in which collaborations are involved afterward. At the start time, a car insurance company probably only involves a group of garages to assess car damages and to repair damaged cars for an insurant, who has bought car insurance from the car insurance company. The insurance company deals with the rest of the issues. More precisely, after the occurrence of a car damage, a process starts, including many interactions among the insurant, a garage and the insurance company (see Figure 1 (a)).

After some time, the insurance company decides to outsource the phone service to a call center. The business process is consequently changed (along the line of Figure 1 (b)). The call center is responsible for registering the insurant information, suggesting an appropriate garage (most time a close by garage is assigned) and notifying the insurance company about the insurant's claim. Except the phone service, the insurance company still needs to handle the rest of services for the insurant. 


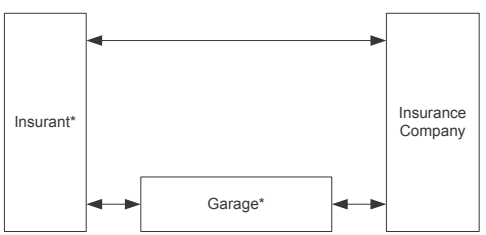

(a) Simple business model

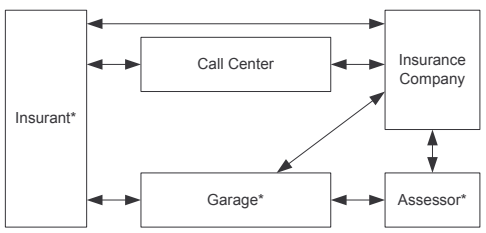

(c) Outsourcing damage assessment

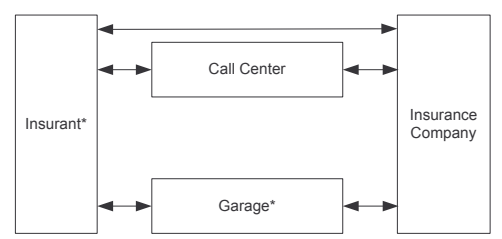

(b) Outsourcing the call center

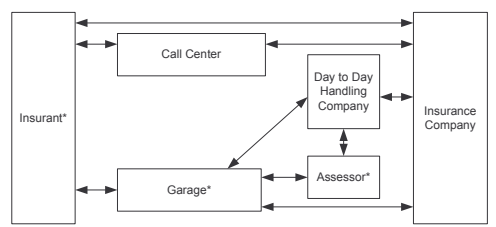

(d) Outsourcing day to day han-

dling

Fig. 1. Car Insurance Business Processes

Continuing it could be an alternative to outsource the inspection of damaged vehicles to an association of assessors. In this business model (see Figure 1 (c)), the assessors conduct the physical inspections of damaged vehicles and agree upon repair figures with the garages. After the call center, the garages and the assessors finish their obligations, the insurance company performs the rest services.

Due to the increasing amount of insurants, the insurance company might finally decide to outsource the daily service to a day to day handling company. The day to day handling company coordinates and manages the operation on a day-to-day level on behalf of the insurance company( see Figure 1 (d)). The detailed obligations of the day to day handling company are provided as follows. After receiving the forward claim from the insurance company, the day to day handling company will agree upon repair costs if an assessor is not required for small damages; otherwise, an assessor will be assigned. After finishing repairs, the garage will issue an invoice to the day to day handling company, which in 
turn will check the invoice against the original estimate. The day to day handling company returns all invoices to the insurance company monthly. As a result the workload of the insurance company is significantly reduced.

Changes of the business models do not necessarily go through from Figure 1(a) to (b), then from (b) to (c) and finally from (c) to (d). Changes can happen, for example, directly from (a) to (d). Figure 1 demonstrates that how a business process is collaborated by more business parties in different circumstances. It also shows some essential characters of multi-party collaborations. One of them is that it is critical to understand when and who did, is doing or will do what in a multiple parties involved business process.

\section{Multi-party Collaboration Modeling Language}

In the business domain, we need to provide detailed and precise descriptions of multi-party business collaborations. In order to represent construct in the business domain, a language for modeling multi-party collaborations should be sufficiently expressive to represent a multi-party collaboration:

- in terms of its structure: who are the parties involved, and how are they interconnected,

- in terms of the commitments associated with those parties,

- and in terms of its processes: what actions are performed by which parties after which properties are satisfied.

An overview of the basic modeling concepts and their relationships is given as a metamodel in Figure 2. A multi-party collaboration consists of roles, parties, channels, commitment, actions and parameters. Parties perform different roles, fulfill different commitments and have many parameters. The roles perform ac- 
tions. A channel connects two or more parties. A commitment aggregates many actions.

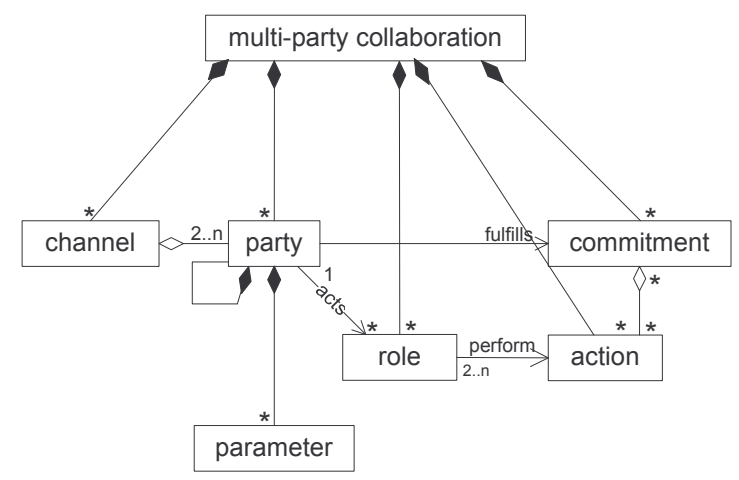

Fig. 2. Metamodel for Multi-party Collaboration Model

\subsection{Collaboration Structure Model}

Collaborated parties involved in a business process are modeled in collaboration structure model. Depending on the chosen scope of the model, parties may represent individual people, organizational units, such as departments, or an entire organization. Furthermore, a party can perform certain commitments.

Parties interact via channels, through which they may exchange information, goods or money. Channels are characterized by a medium (such as Internet, public switched telephone network (PSTN)), by transport (e.g. post, shipping or other ways). Figure 3 shows business parties collaboration model. It depicts a part of the business collaboration consisting of the insurance company and its co-operators.

Modeling collaboration structure is useful to identify the parties involved in business collaboration. It also provides a further step to clarify the responsibilities of parties. 


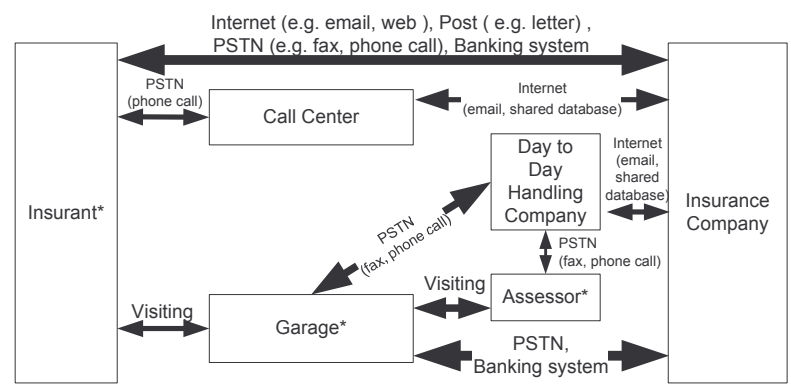

Fig. 3. Business Parties Related by Channels

\subsection{Modeling Commitments between Parties}

To model commitments between multi-parties, we provide speech act theory and its extension in Section 3.2 and define commitments and a commitment model in Section 3.2

Determining the Responsibilities of Parties Part of Austin's work on speech act theory [1], is the observation that utterances are not implied propositions that are true or false, but attempts on the part of the speaker that succeed or fail. Perfomatives, acts, or actions are organized as speech acts and non-speech acts. An individual speech act is either a solicit, which explains an attempt to achieve mutual belief with the addressee that the sender wants the addressee to perform an act relative to the sender's wanting it done, or an assert, which expresses an attempt to achieve mutual belief with the addressee that the asserted statement is true.

The model of speech acts and repartee developed by Longacre recognizes two kinds of relations among successive utterances: replay and resolution in [2] and another two kinds of relations: resolves and completes by Van Dyke Parunakin in [3]. In the model of speech acts and repartee, every utterance in a conversation except for the first must "respond", "reply", "resolve" or "complete" to 
another, otherwise there would be no conversation. Analyzing relations between utterances some characters can be split [4].

In the business process domain physical actions and messages convey information between participants. An initial proposal can be triggered by a certain action and later on be finished by another action. During a multi-party business collaboration, several proposals are initiated by different business parties. Each of them is followed and eventually finished by some action. Actions are thus sorted into different commitments.

For example, based on the collaboration structure model in Figure 3, an insurant phones a call center for a claim. Action A_phoneClaim triggered a conversation between the insurant and the call center to deal with the claim. Actions A_sendlnfo and A_assignGarage follow, and action A_notifyClaim finishes the conversation between the insurant and the call center. Actions A_phoneClaim, A_sendlnfo, A_assignGarage and A_notifyClaim are sorted within a commitment which records obligations of the call center.

Commitments In this paper, a commitment is a guarantee by one party towards another party that some action sequences shall be executed completely provided that some "trigger", "involve", or "finish" action happens and all involved parties fulfill their side of the transaction [5], [6], [7]. To finish a commitment, more than one party must finish relevant actions.

We continue to use the case of which the collaboration structure model is presented in Figure 3. In Table 1 six commitments are identified according to the model of speech acts and repartee.

A multi-party business collaboration consists of a set of commitments. A collaborating party can thus be involved in different commitments playing different roles and an action may be involved in more than one commitment. 


\begin{tabular}{|c|c|c|c|c|}
\hline Commitment & Classific & $\begin{array}{l}\text { ation of Actions and } \mathrm{Co} \\
\text { Tlnvolve }\end{array}$ & $\begin{array}{l}\text { mmitments } \\
\text { IFinish }\end{array}$ & Labels \\
\hline & A_phoneClaim & & & PS.1 \\
\hline $\begin{array}{l}\text { c_pnoneservice } \\
\text { (PS) }\end{array}$ & & $\begin{array}{l}\text { A_sendlnto } \\
\text { A_assignGarage }\end{array}$ & & $\begin{array}{l}\text { PS.2 } \\
\text { PS.3 }\end{array}$ \\
\hline & & & A_notifyClaim & $\begin{array}{l}\text { PS.4, CF.1, } \\
\text { DS1 }\end{array}$ \\
\hline & A_sendCar & & & RS.1 \\
\hline $\begin{array}{l}\text { C_repairService } \\
\text { (RS) }\end{array}$ & A a rreeRenair & A_estimateRepairCost & & $\begin{array}{l}\text { RS.2 } \\
\text { RS } 3 \text { DS } 7\end{array}$ \\
\hline & A-d gleenepail Lat & & A_repairCar & RS.4, DS.8 \\
\hline C_claimForm & A_notifyClaim & A) & & CF.1, PS.4 \\
\hline (CF) & & A-senaclaimrorm & A_returnClaimForm & $\begin{array}{l}\text { CF. } \\
\text { CF. } 3, \text { PR. } 2 \\
\end{array}$ \\
\hline & A_notifyClaim & & & $\begin{array}{l}\text { DS.1, PS.4, } \\
\text { CF.1 }\end{array}$ \\
\hline C_dailyService & & $\begin{array}{l}\text { A_forwardClaim } \\
\text { A_contactGarage }\end{array}$ & & $\begin{array}{l}\text { DS.2 } \\
\text { DS.3 }\end{array}$ \\
\hline & & A_sendRepairCost & & DS. 4 \\
\hline & & $\begin{array}{l}\text { A_assignAssessor } \\
\text { A_sendNewRepairCost }\end{array}$ & & $\begin{array}{l}\text { DS.5, IC. } \\
\text { DS. } 6,1 \text {, I . } 3\end{array}$ \\
\hline & A repaircar & & A_agreeRepairCar & DS.., RS.3 \\
\hline & & A_sendInvoices & & \\
\hline & & & A_forwardinvoices & DS.10, PR.1 \\
\hline C_inspectCar & A_assignAssessor & A incunettor & & IC. $1, \mathrm{DS} .4$ \\
\hline (IC) & & A_Inspectlar & \begin{tabular}{|l} 
A_sendiNewRepairCost \\
\end{tabular} & IC.3,DS.5 \\
\hline C_payRepairCost & A_forwardlnvoices & & & PR.1, DS.10 \\
\hline$(\mathrm{PR})$ & A_returnClaimform & & A_payRepairCost & \begin{tabular}{|l|l|} 
PR.2, CF.3 \\
PR.3
\end{tabular} \\
\hline
\end{tabular}

Table 1. Commitments, Actions and Action abbreviations

It is difficult to represent commitments graphically. In our commitment model, a party is represented as a rectangle with a name. A node denotes a role which should stay in a rectangle. A commitment is indicated by a set of nodes (or commitment connectors) and a set of arrows.

Commitments can be linked by causality, or-split, and-split, or-join, and andjoin as Figure 4 . The causality relation is represented by an arrow from one node to another node. The "or-split" relation is represented by an empty-diamond, which means that a commitment from a role triggers exactly one of multiple commitments from other roles (see Figure 4(a)). The "and-split" relation is expressed by a solid-diamond, which means that a commitment from a role triggers other multiple commitments (see Figure 4(b)). The "or-join" relation is denoted by an empty-box, which means that one of multiple commitment triggers another commitment (see Figure 4(c)). The "and-join" relation is shown by a solid-box, 
which means that multiple commitments together trigger a commitment (see Figure 4(d)).

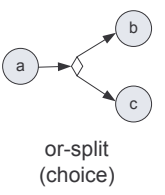

(a)

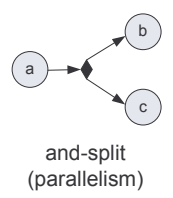

(b)

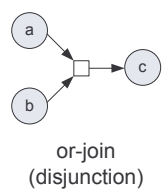

(c)

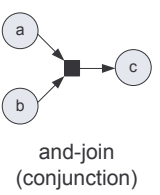

(d)

Fig. 4. Connector Representation

Figure 5 depicts the five parties and six commitments of the case in Figure 3. For example, in party "insurance company", the solid-diamond connects commitments C_phoneService, C_claimForm and C_dailyService. It means that after commitment C_phoneService is fulfilled, both commitments C_claimForm and C_dailyService are triggered. A solid-box is also in party "insurance company", it connects commitments C_dailyService, C_claimForm and C_payRepairCost. It means that commitment C_payRepairCost will be performed after commitments C_dailyService and C_claimForm.

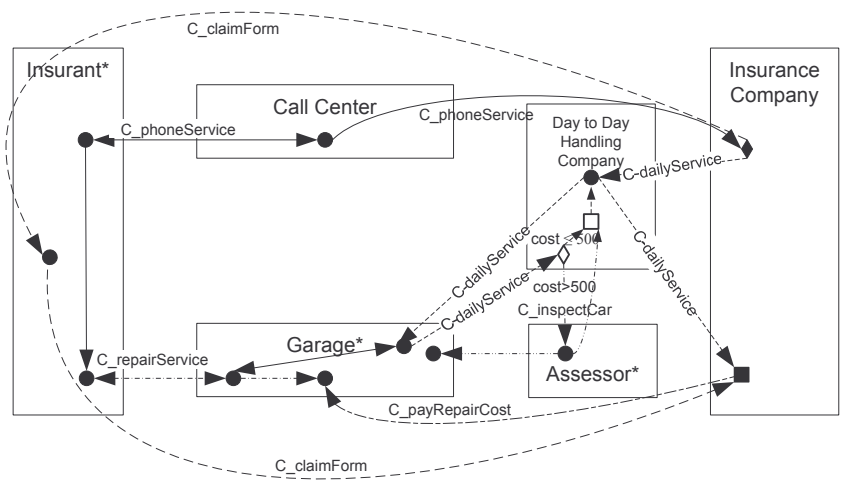

Fig. 5. Commitments of Multi-party Collaboration Model 
In the commitment model, we present the position of each commitment in terms of which parties and roles are involved, and which commitments are triggered, chose and paralleled by other commitments. In the commitment model, we provide an overview of each party's responsibilities. This is very important for both the business side and the IT side as it helps creating a common understanding. In the next section, the behavior of the parties is modeled.

\subsection{Modeling Behaviors of the Parties}

In multi-party business collaboration modeling, behavior is modeled as inter- or intra-organizational business processes. The vertical dimension is the time axis; time proceeds down the page. Each party is represented by a vertical column. An action is the atomic unit of behavior. The causal ordering between actions is modeled by conjunctive and disconjunctive "splits" and "joins".

Each party's behavior is determined by three parameters as found from the business collaboration. The inputs and outputs of a party are domain related. The rules of a party in our model are specified using predicate logic. The input parameter specifies the actions that this party expects to be involved in as object, while the output parameter specifies results of the action. When a party attempts to execute an action, it first checks whether the current input can trigger this action and subsequently generates the output which may be checked against the possible output.

In Figure 6, an action is noted as a table-box where the first column shows the action label which makes it possible to determine the commitment from this action label by looking it up in Table 1; the rest column shows the parameters involved in this action.

The diagram includes a multi-step interaction between participants. It clearly shows which parties will communicate with other parties for which matters. 


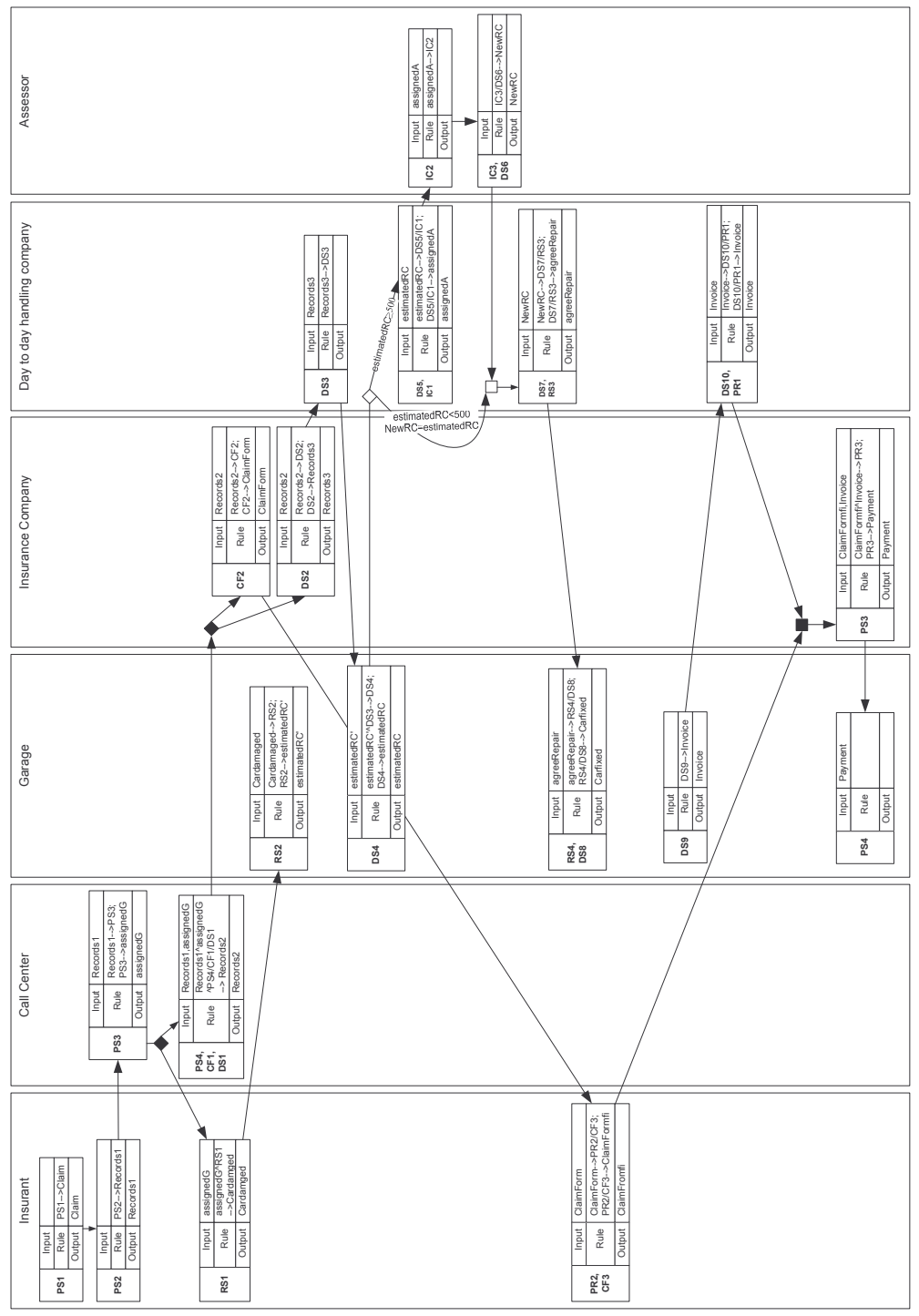

Fig. 6. Interactions between parties

From the insurant's perspective, he only has contact with the call center, the assigned garage and the insurance company. From the insurance company's view, it receives and forwards the claim to the day to day handling company, sends the claim form to the policyholder, and finally pays the repair costs to the garage. 
Each party parameter is also included in the diagram. According to the party properties, each party can determine which actions should or may occur. For example, after having received an input from the party property "Records2", the insurance company will perform actions A_sendClaimForm (as CF2) and A_forwardClaim (as DS2) according to rules "Records2 $\rightarrow \mathrm{CF}_{2}$ " and "Records2 $\rightarrow \mathrm{DS}_{2}$ " respectively that are joined by an "and-join" parameter.

As time passes (from top to down) and satisfying the party parameters, each participant takes actions while the business process is moving forward. A complex multi-party business process is divided into multiple commitments. Furthermore, business process steps are represented as the actions exchanged between the parties.

\section{Related Work}

There are few works concerning multi-party business collaborations. We provide the limitation of UML and Petri net for multi-party business collaboration modeling, as well as other models such as ebXML BPSS, web services choreography and SAP C-Business Scenarios.

According to [8], the UML is not suitable for modeling business. The UML does not support well all the concepts needed for business collaborations. Modeling business collaborations is mainly concerned with what happens at the business level and how it is organized. First, the UML does not support expressing responsibilities. In the business world, parties permit the commitments to each other to execute a business activity. In our approach, the commitment model represents collaborations between business parties and provides the relations of the commitments. Second, for business party behavior, business processes are normally not confined to single actions. Therefore, state diagrams (also for Petri Net) are not really useful here. Most business collaborators do not reason about 
processes in terms of states but rather in terms of the activities performed and results produced. Although UML can be extended with stereotypes and profiles, the stereotyping mechanism just makes it possible to introduce new subtypes of existing concepts and profiles cannot introduce new concepts either [9].

Using the Petri Nets to specify a multi-party business collaboration process, the amount of states of the Petri Net can be significantly increased. Especially, because the multi-party business collaboration process focuses on when and who did, is doing or will do what. A Petri Net representation can be too trivial, even by using state-based workflow patterns [10] because of a big amount of possible combinations of multi-partys behavior.

Two models for ebXML BPSS multi-party collaboration and web services choreography are presented in [11], [12] respectively. Other research [13], [14] on multi-party collaboration tries to break down a multi-party collaboration into a number of bilateral relations. A principle cause behind this is that current e-commerce environments only support bilateral executions. In some simple cases, the approach to support multi-party collaboration execution in current e-commerce environments is to assume the whole business process runs correctly according to a number of bilateral relations. However, in complicated multiparty collaborations this conversion results in information of relations being lost or hidden. Consequently this option to split the multi-party collaborations up into several two-party relations will not work for these complex multi-party collaborations [13], [14].

SAP's collaborative business scenarios describe inter-enterprise business processes from three different perspectives [15], namely business view, interaction view and component view. The purpose of the business view is showing the business advantages of implementing a collaborative business scenario. Business relations per se are out of the scope of our research through. The interaction 
view describes the process design and detailed dependency relationship between the different activities and responsibilities of the participants. It is too simple to describe the relationship like the action relations with conjunctive and disconjunctive "splits" and "joins". The component view describes the logical application components needed to support the business process. Different channels in a collaboration structure model can determine different ways to implement a multi-party collaboration. Commitment model and interaction model provide enough details of interactions between multi-parties. Those three collaboration models can easily map into a component level model by using specifically software implementation packages.

\section{Conclusions}

We have present multi-party business collaboration models from three perspectives. At the collaboration structure model, we provide a view of how business parties are linked. Different links can determine different ways of collaboration. In the commitment model, the responsibilities of all involved parties are presented. Finally, the behavior model provides details of commitment fulfillment. Further research has to map our multi-party business collaboration model to specific implementations like SAP or BAAR' ERP systems. This would allow the semantics of the web of collaborating parties to be validated.

\section{References}

1. Austin, J.: How to do things with words. 2 edn. Oxford University Press (1975)

2. Longacre, R.: An anatomy of speech notions. Lisse: de Ridder (1976)

3. Van Dyke Parunak, H.: Visualizing agent conversations: Using enhanced dooley graphs for agent design and analysis. In: The Second International Conference on Multi-Agent System (ICMAS'96). (1996)

4. Dooley, R.: Repartee as a Graph. Appendix B in [2] (1976)

5. Xu, L., Jeusfeld, M.A.: Pro-active monitoring of electronic contracts. In: The 15th Conference On Advanced Information Systems Engineering in Lecture Notes of Computer Science. Volume 2681., Springer-Verlag (2003) 584-600

6. Xu, L.: Monitoring Multi-party Contracts for E-business. PhD thesis, Tilburg University (2004) 
7. Xu, L., Jeusfeld, M.A.: Detecting violators of multi-party contracts. In: The Conference on CoopIS/DOA/ODBASE in Lecture Notes of Computer Science. Volume 3290., Springer-Verlag (2004) 526-543

8. Steen, M., Lankhorst, M., van de Wetering, R.: Modelling networked enterprises. In: the sixth international Enterprise distributed Object Computing Conference (EDOC'02). (2002)

9. Henderson-Sellers, B.: Some problems with the uml v1.3 metamodel. In: the 34th Annual Hawaii International Conference on Systems Sciences (HICSS-34). (2001)

10. van der Aalst, W., ter Hofstede, A., Kiepuszewski, B., Barros., A.: Workflow patterns. Distributed and Parallel Databases (2003) 5-51

11. Dubray, J.J.: A new model for ebxml bpss multi-party collaborations and web services choreography (2002)

12. Webber, D.: The benefits of ebxml for e-business. In: International Conference on XML (XML'04). (2004)

13. Dubray, J.J.: A new model for ebxml bpss multi-party collaborations and web services choreography (2002) http://www.ebpml.org/ebpml.doc.

14. Haugen, B.: Multi-party electronic business transactions (2002) http://www. supplychainlinks. com/MultiPartyBusinessTransactions. PDF.

15. SAP: mysap.com collaborative business scenarios, whitepaper. In: Walldorf. (2000) 